\title{
Syndecan-4 Is a Receptor for Clathrin-mediated Endocytosis of Arginine-rich Cell-penetrating Peptides
}

Yoshimasa Kawaguchi, ${ }^{\dagger}$ Toshihide Takeuchi, ${ }^{\dagger}$ Keiko Kuwata, ${ }^{\ddagger}$ Junya Chiba, ${ }^{\S}$ Yasumaru Hatanaka, Ikuhiko Nakase, ${ }^{\#}$ and Shiroh Futaki ${ }^{\dagger}, *$

\section{Supporting Information}

Table of Contents

1. Supplementary Figures (SI Figures S1-S7) S-2

2. Supplementary Table (Table S1) 
S-2

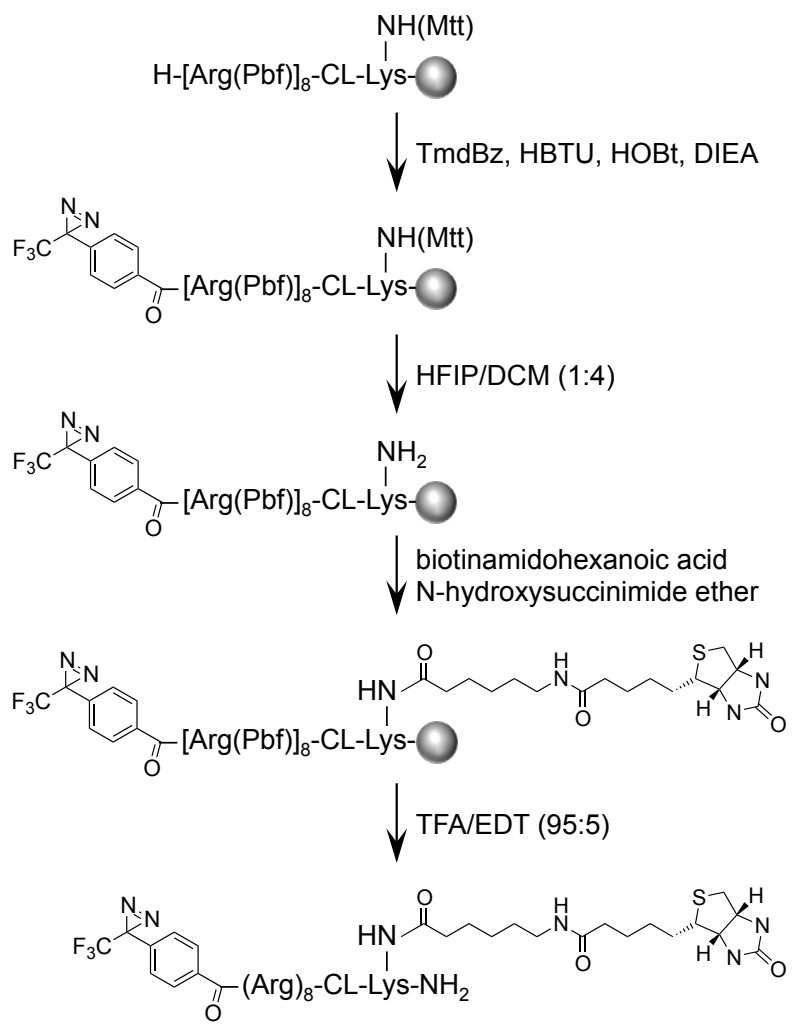

SI Figure S1. Preparation of PhotoR8CL. 
(A)
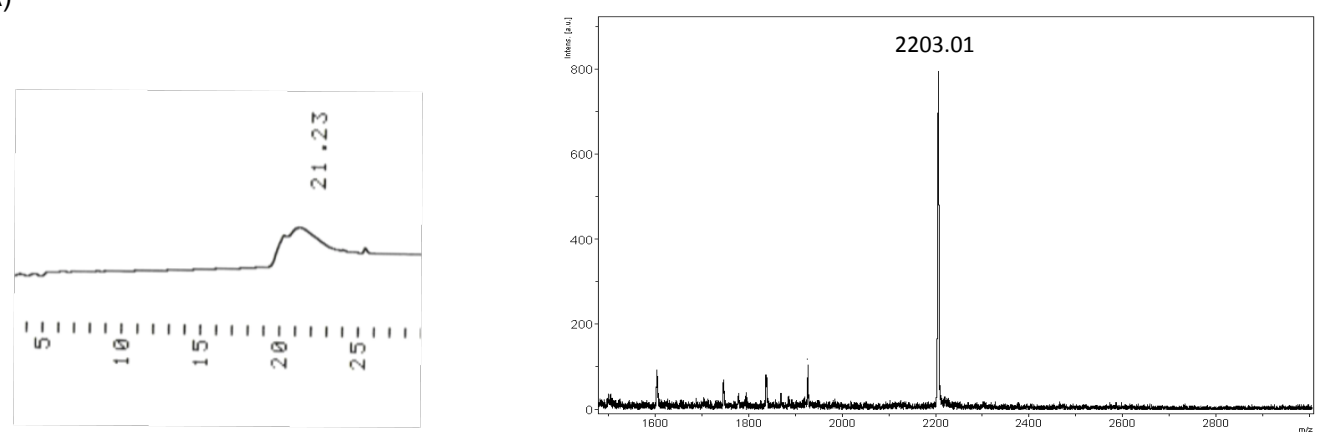

(B)

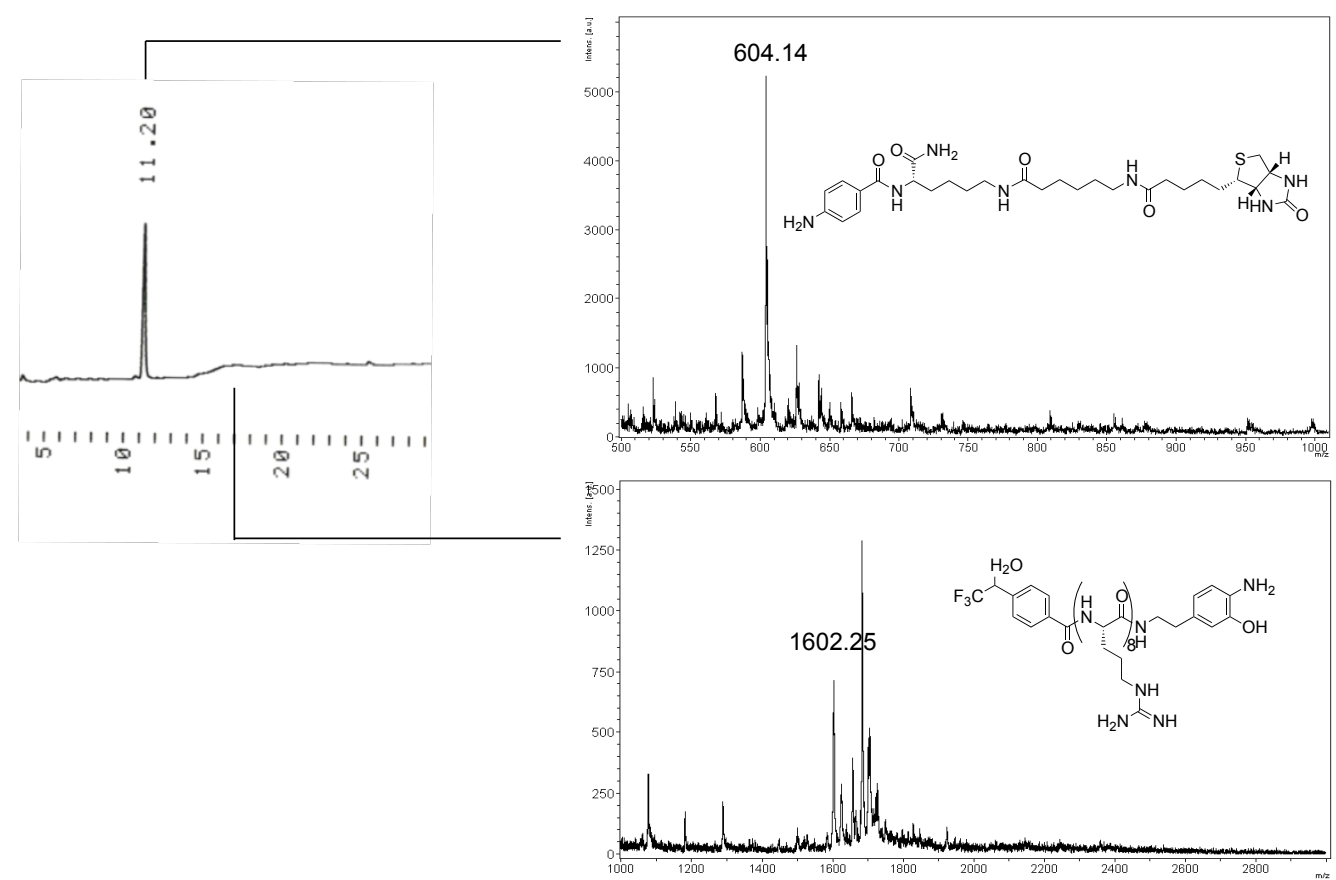

SI Figure S2. Photoreactivity and cleavage activity of PhotoR8CL. (A) HPLC chart (left) and MALDI-TOFMS spectrum (right) of PhotoR8CL that was irradiated at $365 \mathrm{~nm}$ for $10 \mathrm{~min}$. UV irradiation of PhotoR8CL yielded a product with a molecular mass corresponding to PhotoR8CL reacted with $\mathrm{H}_{2} \mathrm{O}$. (B) HPLC chart (left) and MALDI-TOFMS spectra (right) of water-adducted PhotoR8CL that was treated with $\mathrm{Na}_{2} \mathrm{~S}_{2} \mathrm{O}_{4}$ for 15 min. Treatment with $\mathrm{Na}_{2} \mathrm{~S}_{2} \mathrm{O}_{4}$ gave two products corresponding to two fragments derived from PhotoR8CL that would be yielded by reductive cleavage at diazobenzene linker. 


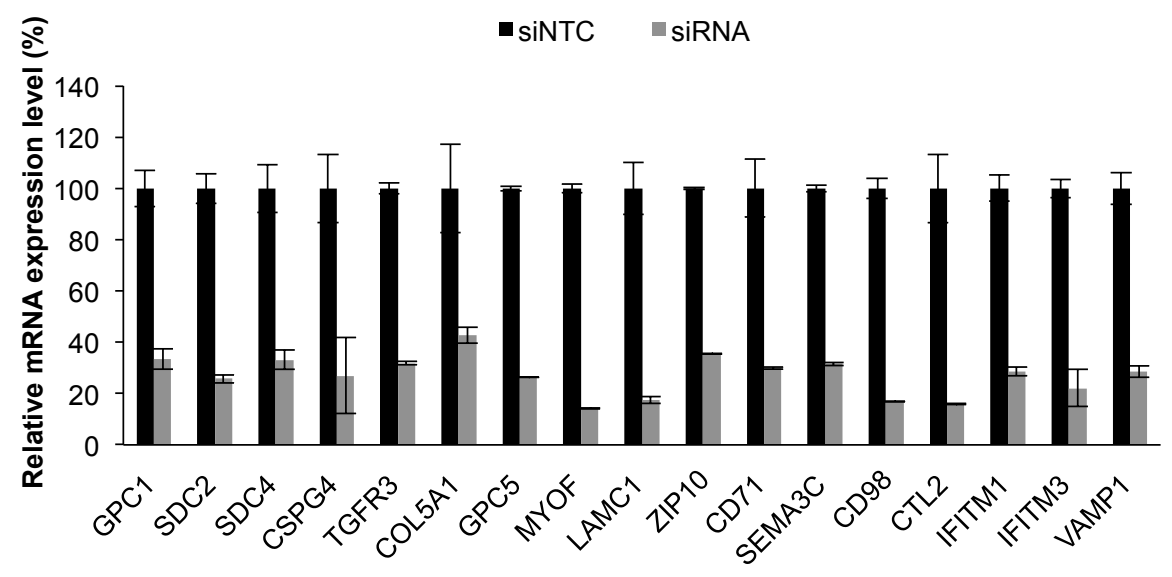

SI Figure S3. Relative mRNA levels analyzed by quantitative RT-PCR. All data are normalized using $\beta$-actin mRNA levels and are represented as a relative value to mRNA levels of siNTC-transfected cells. Data are represented as the mean \pm SD of three independent experiments. siNTC, non-targeting control siRNA. 

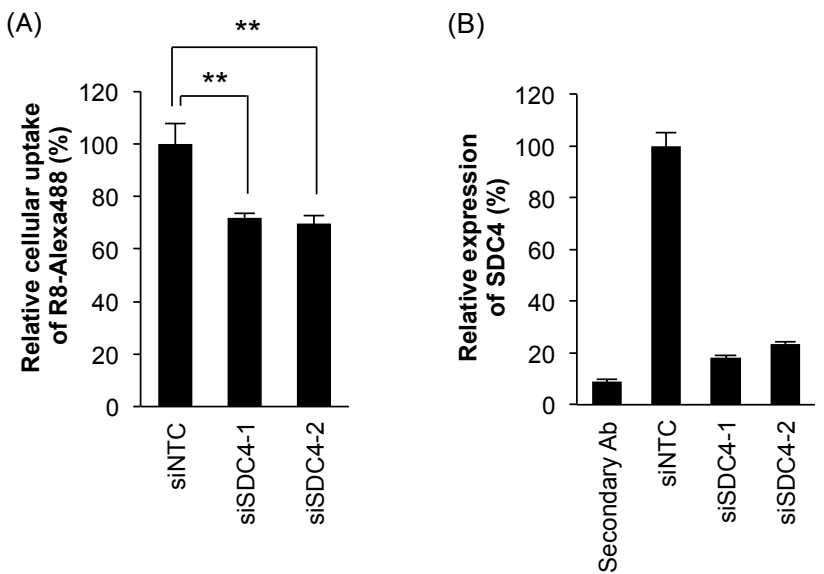

SI Figure S4. Knockdown of syndecan-4 decreases cell-surface expression levels of syndecan-4 and affects R8 internalization. (A) Quantitative analysis of R8 internalization into the syndecan-4-knockdown cells. HeLa cells were transfected with siSDC4s (siSDC4-1 and siSDC4-2), and treated with R8-Alexa488 $(1 \mu \mathrm{M})$ at $37^{\circ} \mathrm{C}$ for $30 \mathrm{~min}$. Data are represented as the mean $\pm \mathrm{SD}$ of three independent experiments $(* * P<0.01$; Student's $t$ test). Note that siSDC4-1 is identical with siSDC4 used in Figures 3-5. (B) Quantitative analysis of cell-surface expression levels of syndecan-4 in the syndecan-4-knockdown cells. HeLa cells were transfected with siSDC4s (siSDC4-1 and siSDC4-2). Cell-surface syndecan-4 was labeled by treatment with anti-syndecan-4 antibody (5G9 mouse monoclonal, Santa Cruz) and anti-mouse IgG conjugated with Alexa488 as secondary antibody, and quantified by flow cytometry. Data are represented as a relative value to cellsurface levels of syndecan-4 in the siNTC-transfected cells. As an experimental control, cells that were treated with secondary antibody alone were used. 
(A)

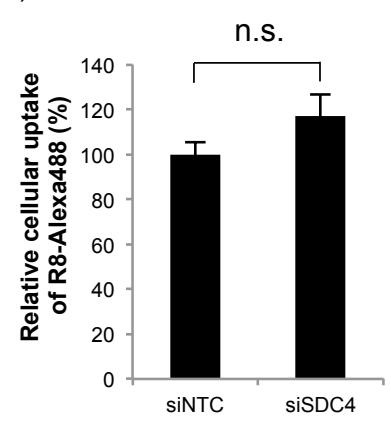

(B)

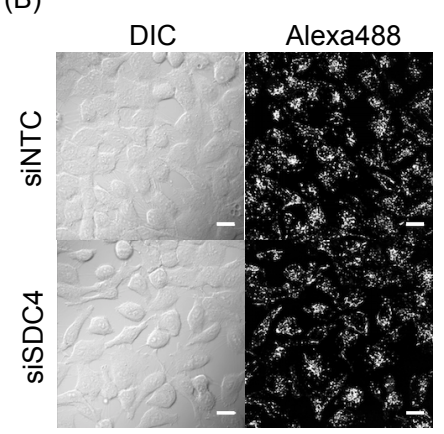

SI Figure S5. Involvement of syndecan-4 in R8 internalization is not observed at relatively high concentration. (A) Bar graph showing R8 internalization levels in the siNTC/siSDC4transfected cells that were treated with $10 \mu \mathrm{M}$ of R8-Alexa488 at $37^{\circ} \mathrm{C}$ for $30 \mathrm{~min}$. n.s., not significant. (B) Confocal microscopic images of the siNTC/siSDC4-transfected cells that were treated as in (A). Knockdown of syndecan-4 had no significant effects on R8 internalization levels under this concentration condition. 
(A)

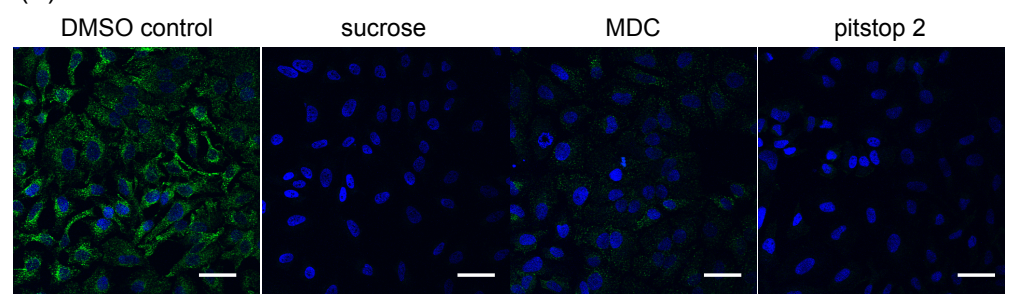

(B)

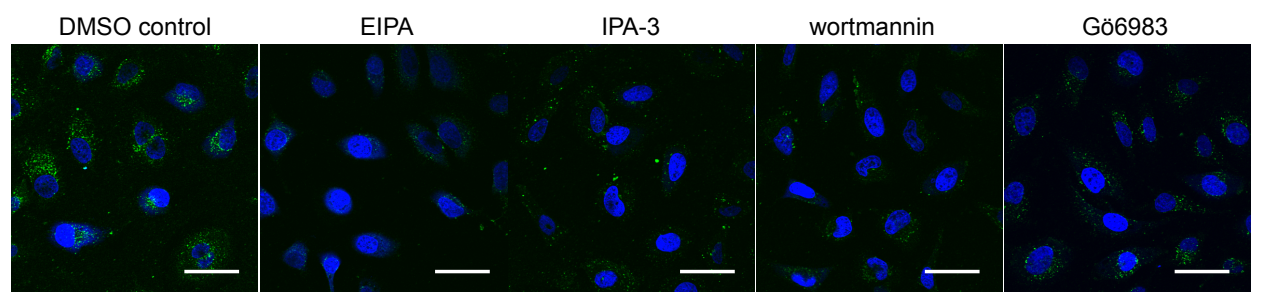

SI Figure S6. Pharmacological inhibitors at the concentrations used in this study inhibit the relevant endocytosis pathways. (A) Confocal microscopic images of the cells treated with transferrin-Alexa488 (Tf-Alexa488), a marker for clathrin-mediated endocytosis, in the presence of inhibitors for clathrin-mediated endocytosis. HeLa cells were pre-treated with sucrose $(0.45 \mathrm{M}), \mathrm{MDC}(50 \mu \mathrm{M})$ or pitstop $2(30 \mu \mathrm{M})$, and incubated with Tf-Alexa488 (25 $\mu \mathrm{g} / \mathrm{mL}$ ) for $10 \mathrm{~min}$ at $37^{\circ} \mathrm{C}$ in the presence of these inhibitors. Surface bound proteins were removed by low $\mathrm{pH}$ wash. The cells were fixed, and subjected to microscopic observation. (B) Confocal microscopic images of the cells that were treated together with $70 \mathrm{kDa}$ dextran-FITC, a marker for macropinocytosis, and epidermal growth factor (EGF) in the presence of inhibitors for macropinocytosis. EGF was used for efficient induction of macropinocytosis. HeLa cells were pre-incubated with EIPA (100 $\mu \mathrm{M})$, IPA-3 $(20 \mu \mathrm{M})$, wortmannin $(500 \mathrm{nM})$ or Gö6983 $(2 \mu \mathrm{M})$, and incubated with $70 \mathrm{kDa}$ dextran-FITC $(1 \mathrm{mg} /$ $\mathrm{mL})$ and $\mathrm{EGF}(500 \mathrm{nM})$ for $1 \mathrm{hr}$ at $37^{\circ} \mathrm{C}$ in the presence of these inhibitors. The cells were then washed and fixed, and subjected to microscopic observation.

In both cases, DMSO was added in the control samples instead of inhibitors. Hoechst 33342 was used for nuclear staining. Scale bar, $50 \mu \mathrm{m}$. 
(A)

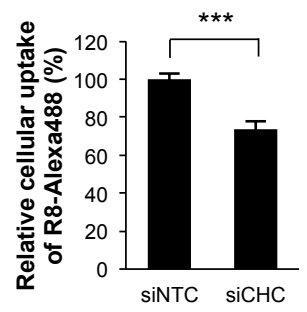

(B)

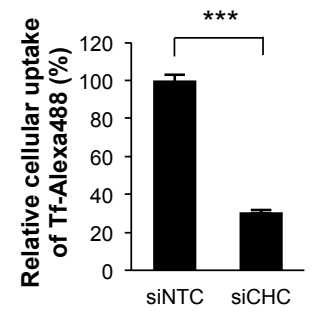

(C)

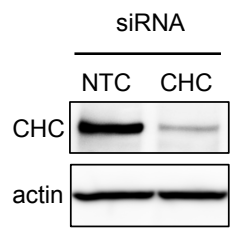

SI Figure S7. RNAi-mediated knockdown of clathrin heavy chain (CHC) decreases R8 internalization. (A) Bar graphs showing levels of R8 internalization in siNTC/siCHCtransfected cells that were treated with $1 \mu \mathrm{M}$ of $\mathrm{R} 8$-Alexa488 at $37^{\circ} \mathrm{C}$ for $10 \mathrm{~min}$. Knockdown of CHC resulted in a decrease in the cellular uptake of R8-Alexa488. (B) Bar graphs showing levels of transferrin (Tf) uptake in the siNTC/siCHC-transfected cells that were treated with $25 \mu \mathrm{g} / \mathrm{mL}$ Tf-Alexa 488 at $37^{\circ} \mathrm{C}$ for $10 \mathrm{~min}$. Knockdown of $\mathrm{CHC}$ resulted in a significant decrease in the cellular uptake of Tf-Alexa488. (C) Immunoblots showing the expression levels of $\mathrm{CHC}$ in the cell transfected with siNTC or siCHC. CHC-specific mouse monoclonal antibody (23/Clathrin Heavy Chain; BD Biosciences) was used. CHC expression level was reduced by RNAi-mediated knockdown. siNTC, non-targeting control siRNA; siCHC, siRNA targeting for CHC. Data are represented as the mean \pm SD of three independent experiments (***P $<0.001$; Student's $t$ test). 
Table S1. Primer sequences for quantitative RT-PCR.

\begin{tabular}{|c|c|c|}
\hline Protein Name & forward primer & reverse primer \\
\hline Glypican-1 & 5'-CTTCCGGGACCTGTACTCAG-3' & 5'-CAGGTAGTCATCAGGCAGCA-3' \\
\hline Syndecan-2 & 5'-GAGTGTATCCTATTGATGACGATGACTAC-3' & 5'-CTCTGGACTCTCTACATCCTCATCAG-3' \\
\hline Syndecan-4 & 5'-GTCTGGCTCTGGAGATCTGG-3' & 5'-CACCAAGGGATGGACAACTT-3' \\
\hline Chondroitin sulfate proteoglycan 4 & 5'-CACACAGAGGAACCCTCGAT-3' & 5'-CTTCAGCGAGAGGAGCACTT-3' \\
\hline Transforming growth factor beta receptor type 3 & 5'-ACA TGG ATA AGA AGC GAT TCA GC-3' & 5'-AAC GCA ATG CCC ATC ACG GTT AG-3' \\
\hline Collagen alpha- $1(\mathrm{~V})$ chain & 5'-GCATTGTCTGTGGTGTGACC-3' & 5'-ATGACTGAACGGCTTGGAAC-3' \\
\hline Glypican-5 & 5'-GGTCGTTGGAAGAACTCTCG-3' & 5'-GCGGCCACAAATCCTATTTA-3' \\
\hline Myoferlin & 5'-GGCTTGCTCTTCACATCCTC-3' & 5'-GGAAAACATCCACCCACATC-3' \\
\hline Laminin subunit gamma-1 & 5'-CAGGCTCCATGAAGCAACAGA-3' & 5'-GCACTTCTCTCACTGTATGTCCCAC-3' \\
\hline Zinc transporter ZIP10 & 5'-TCCTTGCTAGGCGTGATCTT-3' & 5'-TGATCATGTCCACCCTGAGA-3' \\
\hline Transferrin receptor protein 1 & 5'-TCCCAGCAGTTTCTTTCTGTTTT-3' & 5'-CTCAATCAGTTCCTTATAGGTGTCCA-3' \\
\hline Semaphorin-3C & 5'-ATC GCA GCG CTG AGA TTC CTT TAC-3' & 5'-GATGCGCTTGTGTCTCCAGTCC-3' \\
\hline 4F2 cell-surface antigen heavy chain & 5'-CAGGTTCGGGACATAGAGA-3' & 5'-GAGTTAGTCCCCGCAATCAA-3' \\
\hline Choline transporter-like protein 2 & 5'-CTGTGGGATACGTCATGTGC-3' & 5'-TGGACAGGAAGACAGCAGTG-3' \\
\hline Interferon-induced transmembrane protein 1 & 5'-ACTAGTAGCCGCCCATAGCC-3' & 5'-GCACGTGCACTTTATTGAATG-3' \\
\hline Interferon-induced transmembrane protein 3 & 5'-CAAGGAGGAGCACGAGG-3' & 5'-TTGAACAGGGACCAGACG-3' \\
\hline Vesicle-associated membrane protein 1 & 5'-СTCСTCСTTGCCTAATGCAG-3' & 5'-GGACAGAAACCCAGGAATGA-3' \\
\hline
\end{tabular}

